\title{
Numerical Simulation of Hydro- and Magnetohydrodynamic Processes in the Muon Collider Target
}

\author{
Roman Samulyak \\ Center for Data Intensive Computing, Brookhaven National Laboratory, \\ Upton, NY 11973, USA, \\ rosamu@bnl.gov, \\ http://pubweb.bnl.gov/users/rosamu/www/r.samulyak.htm
}

\begin{abstract}
We have developed numerical methods and performed numerical simulations of the proposed Muon Collider target. The target will be designed as a pulsed jet of mercury interacting with strong proton beams in a 20 Tesla magnetic field. A numerical approach based on the method of front tracking for numerical simulation of magnetohydrodynamic flows in discontinuous media was implemented in FronTier, a hydrodynamics code with free interface support. The FronTier-MHD code was used to study the evolution of the mercury jet in the target magnet system. To model accurately the interaction of the mercury target with proton pulses, a realistic equation of state for mercury was created in a wide temperature - pressure domain. The mercury target - proton pulse interaction was simulated during 120 microseconds. Simulations predict that the mercury target will be broken into a set of droplets with velocities in the range $20-60 \mathrm{~m} / \mathrm{sec}$.
\end{abstract}

\section{Introduction}

In order to understand the fundamental structure of matter and energy, an advance in the energy frontier of particle accelerators is required. Advances in high energy particle physics are paced by advances in accelerator facilities. The majority of contemporary high-energy physics experiments utilize colliders. A study group was organized at Brookhaven National Laboratory to explore the feasibility of a high energy, high luminosity Muon-Muon Collider [11]. Such a collider offers the advantages of greatly increased particle energies over traditional electron-positron machines (linear colliders). However, several challenging technological problems remain to be solved. One of the most important is to create an effective target able to generate high-flux muon beams. The need to operate high atomic number material targets, that will be able to withstand intense thermal shocks, has led to the exploration of free liquid jets as potential target candidates for the proposed Muon Collider. The target will be designed as a pulsed jet of mercury (high Z-liquid) interacting in a strong magnetic field with a high energy proton beam $[10,11]$. This paper presents results of numerical studies of hydro- and magnetohydrodynamic (MHD) processes in such a target. 
We have developed an MHD code for multifluid systems based on FronTier, a hydrodynamics code with free interface support. FronTier is based on front tracking $[2,4,5]$, a numerical method for solving systems of conservation laws in which the evolution of discontinuities is determined through solutions of the associated Riemann problems. We also have developed a realistic equation of state for mercury in a wide temperature - pressure domain.

The paper is organized as follows. In Section 2, we describe some details of the Muon Collider target design. In Section 3, we formulate the main system of MHD equations, boundary conditions and discuss some simplifying assumptions used in our numerical schemes. The numerical implementation of the MHD system in the FronTier code is presented in Section 4. Subsection 5 presents simulation results of the mercury target - proton pulse interaction. Subsection 5.2 contains results of the numerical simulation of conducting liquid jets in strong nonuniform magnetic fields and the evolution of the mercury jet in the target solenoid system. Finally, we conclude the paper with the discussion of our results and perspectives for the future work.

\section{Muon Collider Target}

The muon collider target [10] is shown schematically in Figure 1. It will contain a series of mercury jet pulses of about $0.5 \mathrm{~cm}$ in radius and $60 \mathrm{~cm}$ in length. Each pulse will be shot at a velocity of $30-35 \mathrm{~m} / \mathrm{sec}$ into a 20 Tesla magnetic field at a small angle ( $0.1 \mathrm{rad})$ to the axis of the field. When the jet reaches the center of the magnet, it is hit with 3 ns proton pulses arriving with $20 \mathrm{~ms}$ time period; each proton pulse will deposit about $100 \mathrm{~J} / \mathrm{g}$ of energy in the mercury.

The main issues of the target design addressed in our numerical studies are the distortion of the jet due to eddy currents as it propagates through the magnetic coil, the deformation of the jet surface due to strong pressure waves caused by the proton pulses and the probability of the jet breakup. Studying the state of the target during its interaction with proton pulses will help to achieve the maximal proton production rate and therefore an optimal target performance. The target behavior after the interaction with proton pulses during its motion outside the magnet determines the design of the chamber.

\section{Magnetohydrodynamics of Liquid Metal Jets}

The basic set of equations describing the interaction of a compressible conducting fluid flow and a magnetic field is contained in Maxwell's equations and in the equations of fluid dynamics suitably modified $[1,8]$. Namely, the systems contains the mass, momentum and energy conservation equations for the fluid which have hyperbolic nature and a parabolic equation for the evolution of the magnetic field.

$$
\frac{\partial \rho}{\partial t}=-\nabla \cdot(\rho \mathbf{u})
$$




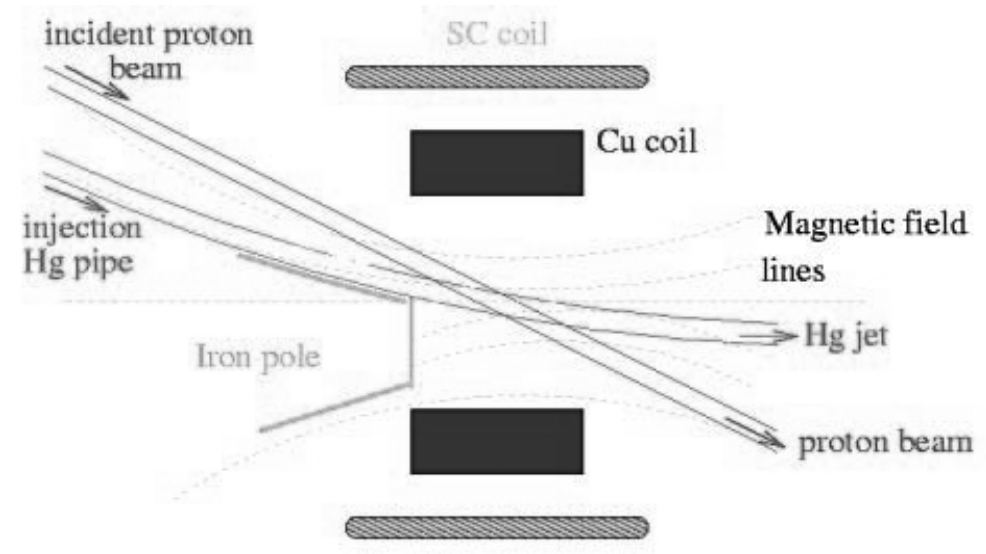

Fig. 1. Schematic of the muon collider target.

$$
\begin{aligned}
\rho\left(\frac{\partial}{\partial t}+\mathbf{u} \cdot \nabla\right) \mathbf{u} & =-\nabla P+\rho \mathbf{X}+\frac{1}{c}(\mathbf{J} \times \mathbf{B}), \\
\rho\left(\frac{\partial}{\partial t}+\mathbf{u} \cdot \nabla\right) U & =-P \nabla \cdot \mathbf{u}+\frac{1}{\sigma} \mathbf{J}^{2}-\frac{1}{c} \mathbf{u} \cdot(\mathbf{J} \times \mathbf{B}), \\
\frac{\partial \mathbf{B}}{\partial t} & =\nabla \times(\mathbf{u} \times \mathbf{B})-\nabla \times\left(\frac{c^{2}}{4 \pi \sigma} \nabla \times \mathbf{B}\right), \\
\nabla \cdot \mathbf{B} & =0,
\end{aligned}
$$

Here $\mathbf{u}, \rho$ and $U$ are the velocity, density and total energy of the fluid, respectively, $P$ is the total stress tensor, $\mathbf{X}$ includes external forces of non-magnetic origin, $\mathbf{B}$ is the magnetic field induction, $\mathbf{J}$ is the current density distribution and $\sigma$ is the fluid conductivity. The magnetic field $\mathbf{H}$ and magnetic induction $\mathbf{B}$ are related by the magnetic permeability coefficient $\mu: \mathbf{B}=\mu \mathbf{H}$.

The system (1-4) must be closed with an equation of state. Equation of state models for the jet material and the ambient gas are discussed in Section 5 .

The following boundary conditions must be satisfied at the jet surface:

i) the normal component of the velocity field is continuous across the material interface.

ii) the normal and tangential components of the magnetic field at the material interface are related as

$$
\begin{gathered}
\mathbf{n} \cdot\left(\mathbf{B}_{2}-\mathbf{B}_{1}\right)=0, \\
\mathbf{n} \times\left(\mathbf{H}_{2}-\mathbf{H}_{1}\right)=\frac{4 \pi}{c} \mathbf{K},
\end{gathered}
$$

where $\mathbf{K}$ is the surface current density. The above jump conditions define the refraction of magnetic lines on the material interface. We can assume $\mu=1$ for most fluids. Notice that the surface current density $\mathbf{K}$ corresponds to a current localized in a thin fluid boundary layer ( $\delta$-functional current) which is nonzero only for superconducting materials. The current density in fluids at normal 
conditions is distributed in the $3 \mathrm{D}$ volume and $\mathbf{K}=0$. Therefore, the equations $(6,7)$ simply require the continuity of the normal and tangential components of the magnetic field.

The behavior of a fluid in the presence of electromagnetic fields is governed to a large extent by the magnitude of the conductivity. For fluid at rest (4) reduces to the diffusion equation

$$
\frac{\partial \mathbf{B}}{\partial t}=\frac{c^{2}}{4 \pi \mu \sigma} \Delta \mathbf{B}
$$

This means that an initial configuration of magnetic field will decay with typical diffusion time

$$
\tau=\frac{4 \pi \mu \sigma L^{2}}{c^{2}}
$$

where $L$ is a characteristic length of the spatial variation of $\mathbf{B}$. Despite being good enough conductors, most of liquid metals including mercury are characterized by small diffusion times (33 microseconds for a mercury droplet of $1 \mathrm{~cm}$ radius) compared to some solid conductors (1 sec for a copper sphere of $1 \mathrm{~cm}$ radius). Therefore the magnetic field penetration in such liquid conductors can be considered as an instantaneous process.

Another crucial phenomena for MHD flows of compressible conducting fluids is the propagation of Alfven waves. For mercury at room temperature the Alfven velocity

$$
\mathbf{v}_{A}=\frac{\mathbf{B}_{0}}{\sqrt{4 \pi \rho_{0}}},
$$

where $\mathbf{B}_{0}$ and $\rho_{0}$ are unperturbed (mean) values of the magnetic induction and density of the fluid, respectively, is $\left[B_{0}\right.$ (Gauss) $\left./ 13.1\right] \mathrm{cm} / \mathrm{sec}$. This is a small number compared with the speed of sound of $1.45 \times 10^{5} \mathrm{~cm} / \mathrm{sec}$ even for the magnetic field of $20 \mathrm{~T}$. In many cases, however, it is not desirable to compute Alfven waves explicitly in the system. If, in addition, both the magnetic field diffusion time and the eddy current induced magnetic field are small, an assumption of the constant in time magnetic field can be made. The current density distribution can be obtained in this case using Ohm's law

$$
\mathbf{J}=\sigma\left(-\operatorname{grad} \phi+\frac{1}{c} \mathbf{u} \times \mathbf{B}\right) .
$$

Here $\phi$ is the electric field potential. The potential $\phi$ satisfies the following Poisson equation

$$
\Delta \phi=\frac{1}{c} \operatorname{div}(\mathbf{u} \times \mathbf{B}),
$$

and the Neumann boundary conditions

$$
\left.\frac{\partial \phi}{\partial \mathbf{n}}\right|_{\Gamma}=\frac{1}{c}(\mathbf{u} \times \mathbf{B}) \cdot \mathbf{n},
$$

where $\mathbf{n}$ is a normal vector at the fluid boundary $\Gamma$. This approach is applicable for the study of a liquid metal jet moving in a strong magnetic field. 
We shall use also the following simplification for the modeling of a thin jet moving along the solenoid axis. Let us consider a ring of liquid metal of radius $r$ that is inside a thin jet moving with velocity $u_{z}$ along the axis of a solenoid magnet. The magnetic flux $\Phi=\pi r^{2} B_{z}$ through the ring varies with time because the ring is moving through the spatially varying magnetic field, and because the radius of the ring is varying at rate $u_{r}=d r / d t$. Therefore, an azimuthal electric field is induced around the ring:

$$
\begin{aligned}
2 \pi r E_{\phi}=-\frac{1}{c} \frac{d \Phi}{d t} & =-\frac{\pi r^{2}}{c} \frac{d B_{z}}{d t}-\frac{2 \pi r u_{r} B_{z}}{c} \\
& =-\frac{\pi r^{2} u_{z}}{c} \frac{\partial B_{z}}{\partial z}-\frac{2 \pi r u_{r} B_{z}}{c} .
\end{aligned}
$$

This electric field leads to an azimuthal current density

$$
J_{\phi}=\sigma E_{\phi}=-\frac{\sigma r u_{z}}{2 c} \frac{\partial B_{z}}{\partial z}-\frac{\sigma u_{r} B_{z}}{c},
$$

which defines the Lorentz force in the momentum equation (2) and leads to the distortion of the jet moving in a non-uniform magnetic field.

The linear stability analysis of thin conducting liquid jets moving along the axis of a uniform magnetic field [1] and the corresponding analysis for the Muon Collider target [6] show that an axial uniform field tends to stabilize the jet surface. The influence of a strong nonuniform field is studied below by means of the numerical simulation.

\section{Numerical Implementation}

In this section, we shall describe numerical ideas implemented in the FronTier MHD code. FronTier represents interfaces as lower dimensional meshes moving through a volume filling grid [4]. The traditional volume filling finite difference grid supports smooth solutions located in the region between interfaces. The location of the discontinuity and the jump in the solution variables are defined on the lower dimensional grid or interface. The dynamics of the interface comes from the mathematical theory of Riemann solutions, which is an idealized solution of a single jump discontinuity for a conservation law. Where surfaces intersect in lower dimensional objects (curves in three dimensions), the dynamics is defined by a theory of higher dimensional Riemann problems such as the theory of shock polars in gas dynamics. Nonlocal correlations to these idealized Riemann solutions provide the coupling between the values on these two grid systems.

The computation of a dynamically evolving interface requires the ability to detect and resolve changes in the topology of the moving front. A valid interface is one where each surface and curve is connected, surfaces only intersect along curves and curves only intersect at points. We say that such an interface is untangled. Two independent numerical algorithms, grid-based tracking and grid-free tracking, were developed $[4,5]$ to resolve the untangling problem for 
the moving interface. The advantages and deficiencies of the two methods are complementary and an improved algorithm combining them into a single hybrid method was implemented in the FronTier code and described in [5].

We solve the hyperbolic subsystem of the MHD equations, namely the equations (1-3), on a finite difference grid in both domains separated by the free surface using FronTier's interface tracking numerical techniques. Some features of the FronTier hyperbolic solvers include the use of high resolution methods such as MUSCL, Godunov and Lax-Wendroff with a large selection of Riemann solvers such as the exact Riemann solver, the Colella-Glaz approximate Riemann solver, the linear US/UP fit (Dukowich) Riemann solver, and the Gamma law fit. We use realistic models for the equation of state such as the polytropic and stiffened polytropic equation of state, the Gruneisen equation of state, and the SESAME tabular equation of state.

The evolution of the free fluid surface is obtained through the solution of the Riemann problem for compressible fluids $[4,9]$. Notice that since we are primarily interested in the contact discontinuity propagation, we do not consider the Riemann problem for the MHD system and therefore neglect elementary waves typical for MHD Riemann solutions.

We have developed an approach for solving the equation (4) for the magnetic field evolution and the Poisson equation (10) using a mixed finite element method. The existence of a tracked surface, across which physical parameters and the fluid solution change discontinuously, has important implications for the solution of an elliptic or parabolic system by finite elements. Strong discontinuities require that the finite element mesh align with the tracked surface in order not to lose the resolution of the parameter/solution. A method for the generation of finite element mesh conforming to the interface and scalable elliptic/parabolic solvers will be presented in a forthcoming paper.

The aim of the present MHD studies is the numerical simulation of free thin liquid metal jets entering and leaving solenoid magnets using the abovementioned simplifying assumptions. The eddy current induced magnetic field in such problem is negligible compared to the strong external magnetic field. We applied the approximation $d \mathbf{B} / d t=0$ and implemented the MHD equations (13 ) in FronTier's hyperbolic solvers using the approximate analytical expression (11) for the current density distribution.

\section{Results of Numerical Simulation}

\subsection{Interaction of Mercury Jet with Proton Pulses}

Numerical simulations presented in this section aid in understanding the behavior of the target under the influence of the proton pulse, and in estimating the evolution of the pressure waves and surface instabilities. We have neglected the influence of the external magnetic field on the hydrodynamic processes driven by the proton energy deposition.

The influence of the proton pulse was modeled by adding the proton energy density distribution to the internal energy density of mercury at a single time 
step. The value and spatial distribution of the proton energy was calculated using the MARS code [10].

To model accurately the interaction of the mercury target with proton pulses, a tabulated equation of state for mercury was created in a wide temperature pressure domain which includes the liquid-vapor phase transition and the critical point. The necessary data describing thermodynamic properties of mercury in such a domain were obtained courtesy of T. Trucano of Sandia National Laboratory. This equation of state was not directly used in the code for current simulations but it provided necessary input parameters for the stiffened polytropic eos model [9].

The evolution of the mercury target during $120 \mathrm{mks}$ due to the interaction with a proton pulse is shown in Figure 2. Simulations predict that the mercury target will be broken into a set of droplets with velocities in the range $20-60$ $\mathrm{m} / \mathrm{sec}$. The pressure field inside the jet developed small regions of negative pressure which indicates a possibility for the formation of cavities. Detailed studies of the cavitation phenomena in such a target will be done using the original tabulated equation of state for mercury.

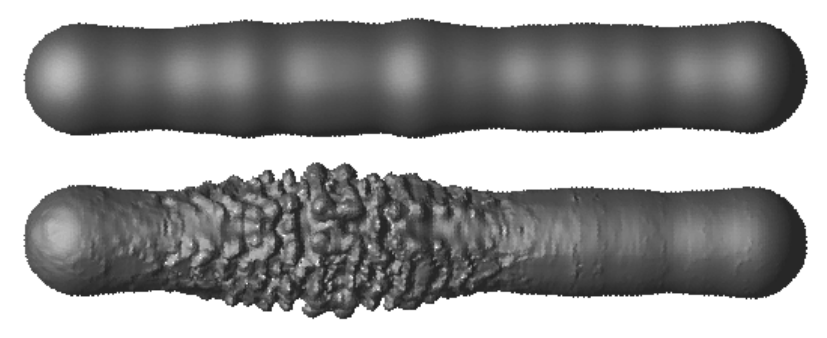

Fig. 2. Evolution of the target during 120 mks driven by the proton energy deposition.

\subsection{Motion of Liquid Metal Jets in Magnetic Fields}

In this section, we shall present numerical simulation results of thin jets of conducting fluid in highly nonuniform magnetic fields. In this numerical experiment, a $1 \mathrm{~cm}$ radius liquid jet is sent into a $20 \mathrm{~T}$ solenoid with the velocity $90 \mathrm{~m} / \mathrm{sec}$ along the solenoid axis. The density of the liquid is $1 \mathrm{~g} / \mathrm{cm}^{3}$, the electric conductivity is $10^{16}$ in Gaussian units, and the initial pressure in the liquid is 1 atm. The electrically and magnetically neutral gas outside the jet has density 0.01 $\mathrm{g} / \mathrm{cm}$ and the same initial pressure. The thermodynamic properties of the ambient gas were modeled using the polytropic equation of state [3] with the ratio of specific heat $\gamma=1.4$ and the ideal gas constant $R=1$. The properties of the liquid jet were modeled using the stiffened polytropic equation of state with the Gruneisen exponent $=5$ and the stiffened gas constant $P_{\infty}=3 \cdot 10^{9} \mathrm{~g} /\left(\mathrm{cm} \cdot \mathrm{sec}^{2}\right)$. 
The field of a magnetic coil of rectangular profile and $8 \times 8 \times 20 \mathrm{~cm}$ size was calculated using exact analytical expressions.

A set of images describing the evolution of the liquid jet as it enters and leaves the solenoid is depicted in Figure 3. The strong nonuniform magnetic field near the solenoid entrance squeezes and distorts the jet. The magnetic field outside the solenoid stretches the jet which results in the jet breakup. Notice that the expression (11) is not valid for an asymmetrically deformed jet as well as for a system of liquid droplets. The simulation looses the quantitative accuracy when the jet is close to the breakup. The numerical algorithm based on finite elements conforming to the moving interface described briefly in Section 3 is designed for solving accurately the equation for the current density distribution and magnetic field evolution. The numerical simulation of the jet breakup phenomena using such approach will be presented in a forthcoming paper.

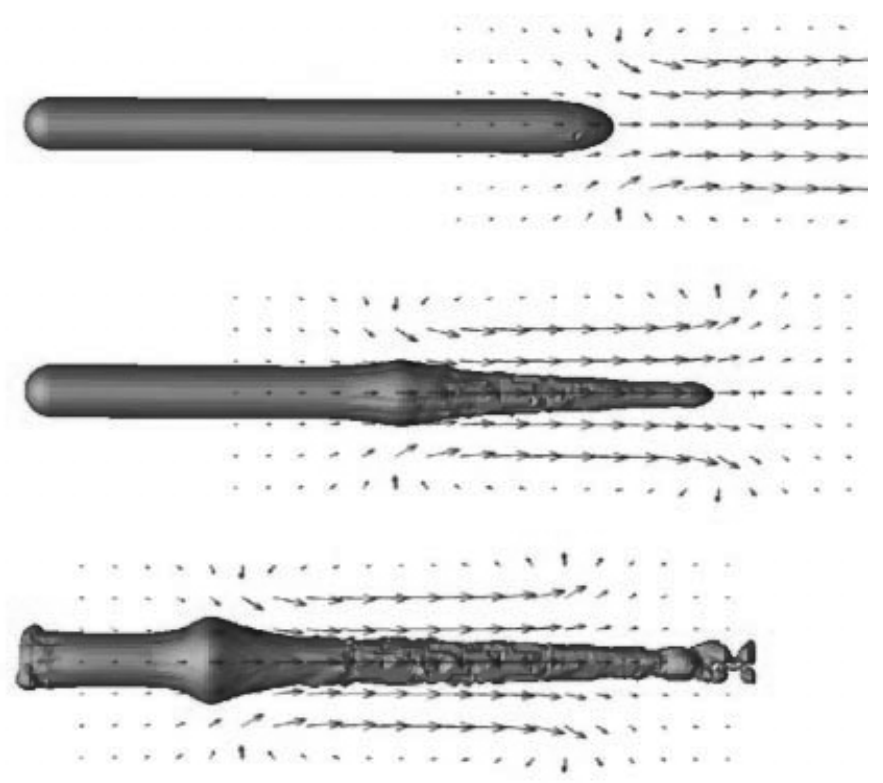

Fig. 3. Liquid metal jet in a $20 \mathrm{~T}$ solenoid.

The numerical simulation demonstrates the influence of strong magnetic field gradients on the jet distortion. To avoid such instabilities of the mercury target during its propagation in a $20 \mathrm{~T}$ magnetic field, a special magnet system was designed [10]. The nozzle of the mercury target was placed inside the main 20 $\mathrm{T}$ resistive magnetic coil. Therefore the jet will not meet strong magnetic field gradients before the interaction with the proton pulses. The magnetic field of superconducting and matching solenoids, needed for carrying muons to the linear accelerator (RF linac), essentially reduce the magnetic field gradient outside the 
main solenoid. Therefore the motion of the mercury target between the nozzle and the mercury dump is within a region of an almost uniform magnetic field.

We performed numerical simulations of the mercury jet motion in such magnetic system. Our results show that the magnetic field influence will not lead to significant jet deformations. However, the pressure waves, caused by the magnetic field forces, which propagate mainly along the jet axis speedup surface instabilities of the jet.

\section{Conclusions}

In this paper, we described a numerical approach based on the method of front tracking for the numerical simulation of magnetohydrodynamic free surface flows. The method was implemented in FronTier, a hydrodynamics code with free interface support. The FronTier MHD code was used for the numerical simulation of thin conducting liquid jets in strong nonuniform magnetic fields. Our results demonstrate a big influence of the Lorentz force on the stability of jets. The Loerntz force distorts a jet and stimulates the jet breakup in the region of nonuniform magnetic field behind a solenoid.

The method was also used for numerical simulation of the muon collider target. The magnetic field of the solenoid system designed for the muon collider experiments slowly decreases behind the main $20 \mathrm{~T}$ solenoid. This allows the mercury target to leave the interaction chamber without significant distortions. However, the magnetic forces speedup the natural instability and the pinchoff of the mercury jet.

We performed also numerical simulations of the interaction of the mercury jet with a proton pulse. Simulations predict that the mercury target will be broken into a set of droplets with velocities in the range $20-60 \mathrm{~m} / \mathrm{sec}$. An accurate analysis of the pressure field inside the jet indicates a possibility for the formation of cavities.

We have developed an algorithm for accurate numerical calculation of the magnetic field evolution and the current distribution in moving domains based on mixed finite elements dynamically conforming to evolving interfaces. Our future goals include studies of the jet breakup phenomena in nonuniform fields, the stabilizing effects of uniform magnetic field on liquid jets, and global numerical simulations of the mercury target including the target - proton beam interaction in the presence of a strong magnetic field.

Acknowledgments: The author is grateful to J. Glimm, K. McDonald, H. Kirk, R. Palmer and R. Weggel for fruitful discussions. Financial support has been provided by the USA Department of Energy, under contract number DEAC02-98CH10886. 


\section{References}

1. Chandrasekhar, S.: Hydrodynamics and Hydrodynamic stability. Clarendon Press, Oxford (1961)

2. Chern, I.R., Glimm, J., McBryan, O., Plohr, B., Yaniv, S.: Front tracking for Gas Dynamics. J. Comp. Phys. 62 (1986) 83-110

3. Courant, R., Friedrichs, K.: Supersonic Flows and Shock Waves. Interscience, New York (1948)

4. Glimm, J., Grove, J.W., Li, X.L., Shyue, K.M., Zhang, Q., Zeng, Y.: Three dimensional front tracking. SIAM J. Sci. Comp. 19 (1998) 703-727

5. Glimm, J., Grove, J.W., Li, X.L., Tan, D.: Robust computational algorithms for dynamic interface tracking in three dimensions. Los Alamos National Laboratory Report LA-UR-99-1780

6. Glimm, J., Kirk, H., Li, X.L., Pinezich, J., Samulyak, R., Simos, N.: Simulation of 3D fluid jets with application to the Muon Collider target design. Advances in Fluid Mechanics III (Editors: Rahman, M., Brebbia, C.), WIT Press, Southampton Boston (2000) $191-200$

7. Kirk, H., et al.: Target studies with BNL E951 at the AGS. Particles and Accelerators 2001, June 18-22 (2001) Chicago IL

8. Landau, L.D., Lifshitz, E.M.: Electrodynamics of Continuous Media. Addison - Wesley Publishing Co., Reading Massachusetts (1960)

9. Menikoff, R., Plohr, B.: The Riemann problem for fluid flow of real materials. Rev. Mod. Phys. 61 (1989) 75-130

10. Ozaki, S., Palmer, R., Zisman, M., Gallardo, J. (editors) Feasibility StudyII of a Muon-Based Neutrino Source. BNL-52623 June 2001; available at http://www.cap.bnl.gov/mumu/studyii/FS2-report.html

11. Palmer, R.B.: Muon Collider design. Brookhaven National Laboratory report BNL65242 CAP-200-MUON-98C (1998) 